\title{
Bilateral Kimura's disease with sinusitis and parotid gland involvement: A case report and a brief review of literature
}

\author{
Priyokumar $\mathrm{OS}^{1}$, Niteshore $\mathrm{MS}^{2}$, Ratan $\mathrm{K}^{3}$. \\ ${ }^{1}$ (Dept. of Otorhinolaryngology, Regional Institute of Medical Sciences, India) \\ ${ }^{2}$ (Dept. of Otorhinolaryngology, Regional Institute of Medical Sciences, India) \\ ${ }^{3}$ (Dept. of Pathology, Regional Institute of Medical Sciences, India)
}

\begin{abstract}
Kimura's disease (KD) is a rare eosinophilic inflammatory disorder of unknown etiology. It presents as a deep, subcutaneous mass in the head and neck region. The important clinicopathologic features is its frequent association with regional lymph node and/or major salivary glands involvement. We report the case of a 27 years male presented with multiple nodular swelling in the bilateral periauricular region with parotid and neck extensions associated with sinusitis. The patient also gave history of frequent sneezing, nasal obstruction, watery rhinorrhoea and heaviness of head suggestive of allergic rhinosinusitis. Multiple fine needle aspiration cytology (FNAC) gave non-specific findings. Biopsy showed typical features of Kimura's disease. The patient was treated with surgical excision of the mass on both sides followed by post-operative steroids and antihistaminics. We report the case of kimura's disease with allergic rhinosinusitis as there is no reports of its association with rhinosinusitis according to our literature search and also because of its rarity and difficulty in diagnosis. A brief review of literature is also presented herewith.
\end{abstract}

Key words: angiolymphoid hyperplasia, antihistaminics, kimura's disease, steroids.

\section{Introduction}

Kimura's disease (KD) is a rare eosinophilic inflammatory disorder of unknown etiology presenting as a deep, subcutaneous mass in the head and neck region and frequently associated with regional lymphadenopathy or salivary gland involvement ${ }^{1}$. Mostly regional lymph node and/or major salivary glands involvement occurs unilaterally however bilateral involvement rarely occurs. But there is hardly any mention of occurrence of sinusitis with Kimura's disease. We report a case of Head and Neck bilateral kimura's disease with allergic rhinosinusitis, because of its rarity and difficulty in diagnosis. A brief review of literature is also presented to highlight this rare but frustrating disease.

\section{Case History}

A 27 years male presented with multiple nodular swelling in the bilateral periauricular region with neck extensions. There was also an isolated nodular mass in the suboccicular region on the right with a dimension of $13 \mathrm{~cm} \mathrm{X} 11 \mathrm{~cm}$, whereas on the left it was $12 \mathrm{~cm} \mathrm{X} 10 \mathrm{~cm}$ [Figure 1]. Scar tissue was present on both the sides with history of previous surgery elsewhere around 17 months back. The swellings were non-tender with mixed consistencies- firm over the nodules but soft in between the nodules. The patient also complained of itching in and around the swellings with visible scratch marks. The patient also gave history of frequent sneezing, nasal obstruction, watery rhinorrhoea and heaviness of head suggestive of allergic rhinosinusitis. Routine investigation shows significant peripheral eosinophillia $\left(1.8 \times 10^{9} / \mathrm{L}\right)$ and $21 \%$ in differential WBC count with mild elevation of the ESR. CT scan of the Paranasal sinuses showed features of bilateral maxillary and ethmoidal sinusitis with large lobulated inhomogeneous contrast enhancing soft tissue mass lesion involving both superficial and deep lobe of both the parotid glands [figure 2,3]. The sub-mandibular glands were not involved. Laboratory investigations showed no sign of renal involvement. Multiple FNAC gave findings of nonspecific reactive lymphadenitis. A decision was made to operate and remove the involved lymph nodes and the masses from one side and perform a histopathological examination of the excised specimen. Biopsy showed subcutaneous tissue with striking lobulated appearance of lymphoid and eosinophils separated by fibrous stroma. Endothelial cell proliferation was not conspicuous and no parasite or any granuloma were seen. Section from the lymph nodes attached to the mass showed capsular thickening, fibrosis and follicular hyperplasia having eosinophils inside the lymphoid follicles and eosinophillic microabscess at the interfollicular region [Figure 4,5,6]. The overall features were consistent with the diagnosis of Kimura's Disease.

The patient underwent a second operation to remove the disease from the other side after five months. Intraoperative findings on both the operation include enlarged multiple lymph nodes with a well-defined mass superficial to the parotid glands but on both the occasions, intra-parotid lymph nodes as well as the subcutaneous tissues were involved in both the sides which were excised. The marginal mandibular nerve of the left side had to be sacrificed due to close involvement of the lesion with the nerve. The involved sub-occipital 
lymph nodes were also excised. The patient was put on high dose steroid therapy, antihistaminics and monteleukast post-operatively. Radiotherapy or chemotherapeutic agents were not used in the management keeping in mind the young age of the patient.

\section{Discussion}

Kimura disease is considered much more prevalent in young males of Asian lineage. There is a marked predominance of male patients; the male/female ratio is 2:1-5:1 and the onset of the disease occurs mostly in the third decade of life[1,2,3].

The disease is characterized by a triad of unpainful subcutaneous masses in the head and neck, eosinophilia in the peripheral blood and in tissues, and marked increase in serum levels of immunoglobulin $\mathrm{E}$ (IgE). The nodular lesions are deep-seated in subcutaneous tissue and clinically may mimic a neoplasm. One of the important clinicopathologic features is its frequent association with regional lymph node and/or major salivary glands involvement $[1,2,3,4]$

In a study on Kimura's disease in children by Vishwantha, all patients presented with head and neck swelling without upper limb involvement. Fifty per cent of these patients had post auricular involvement. Kimura's disease involving upper limb and axillary lymph node is also reported. Involvement of the groin region and limbs is very rare. Lesions in other areas were also described such as the inguinal area, upper limbs and chest wall. Indeed, isolated lymphadenopathy might be the only initial presentation of Kimura disease $[4,7,8]$.

Renal involvement is the only systemic manifestation which may affect up to $60 \%$ of patients as membranous glomerulonerphritis, minimal change glomerulonephritis, diffuse proliferative glomerulonephritis, mesangial proliferative glomerulonephritis, IgA nephropathy and also nephritic syndrome (12\% of cases). Most common renal involvement occurs as membranous glomerulonephritis[5,6].

Its nearest differential diagnosis is Angiolymphoid hyperplasia with eosinophilia. The association with parotid gland leads to the final diagnosis of Kimura's disease. The histopathological changes in KD consist of a massive, nodular, diffuse and mixed inflammatory infiltrate composed mainly of lymphocytes and eosinophils, occupying all the extension of reticular dermis, subcutaneous tissue and, sometimes, the muscle fascia and the skeletal muscle. The inflammatory infiltrate is poorly circumscribed and contains numerous lymphoid follicles and infiltration of adjacent salivary glands may also occur. Lymphoid follicles are hyperplastic and contain prominent germination centers . Eosinophilic infiltration may occur occasionally with areas of necrosis and eosinophillic microabscess. At larger magnifications, Angiolymphoid hyperplasia with eosinophilia is characterized by enlarged and sometimes polygonal endothelial cells with prominent vacuoles in the cytoplasm. These vacuoles are not present in Kimura disease. Although eosinophils are present in both diseases, they may be in a small number or practically absent in Angiolymphoid hyperplasia with eosinophilia, but they are always abundant in Kimura disease. Lastly, while the lymphoid follicles are absolutely necessary for the diagnosis of Kimura disease, they are present in less than $10 \%$ of cases of Angiolymphoid hyperplasia with eosinophilia[1,2]

The pathophysiology of Kimura disease remains unknown, although allergic reaction, trauma and autoimmune process have been implicated as triggering factors. A number of theories about its origin have been proposed which include interference with immune regulation, atopic reaction to a continuos antigenic stimulus (especially Candida albicans) parasitic infection and neoplasm. The most popular theory is that of Candida acting as a source of persistent antigenemia, although neither hyphae nor spores have been isolated $[1,2,5]$.

FNAC is misleading and diagnosis is established only on histopathological examination. ${ }^{5}$ Computed tomography (CT scan) and magnetic resonance imaging (MRI) have been useful in the investigation of the Kimura's disease. A CT scan showed contrast intensity in the neck node. On MRI the involved neck nodes demonstrated high T 1 and T 2 weighted signal intensity. A CT scan is useful in delineating the site of lesion separating it from the surrounding abnormal tissue. It is also important in defining the extent of the disease before any surgical intervention. Ultra-sonographic features are nonspecific. It only can show variable characteristics from pure necrosis to a rather heterogeneous echo texture. Surgical excision is the most common diagnostic measure and is often therapeutic[3].

The optimal treatment of KD is not well defined in the literature. At initial presentation, surgical biopsy is the most frequent diagnostic procedure, and excision may be curative. However, recurrence is common. Complete surgical removal of a head and neck lesion may be difficult given the subsequent facial reconstruction needed and the danger of neurologic deficit such as facial nerve injury in parotidectomy. Complete excision is not always possible due to the extremely diffuse nature of the disease. There have also been reports of recurrence in 15 to $40 \%$ of cases even after apparently adequate surgical excision and excisional biopsies, but this may be because of the poor delineation of the borders of KD, suggesting that adjunctive frozen sections may be useful, especially in reexcision and recurrence. Other therapeutic options including radiation, systemic corticosteroids, cyclosporine A and pentoxyphylline, have all been tried with variable responses. Due to the above postulated pathogenesis, additional medical therapy including regional or systemic steroid therapy, 
cytotoxic therapy and radiation has been utilized. Oral steroid have been the mainstay of therapy for subcutaneous plaque or nodule of KD even though steroid withdrawal can result in lesion recurrences. Steroid therapy is useful to shrink the KD tumor in preparation before surgery. It is also indicated in frequent relapses or cases complicated by nephrotic syndrome. Patients are started on high dose of oral steroid and the dose is tapered to the minimal dose that will suppress the recurrence. Steroid withdrawal can result in lesion recurrence. Long term steroid treatment can have many side effects that limit its use. Considering the benign nature of KD, radiation should be reserved as a second line treatment[9].

Local initial recurrence can often be managed by local excision. If recurrence becomes frequent or patient developed symptomatic nephrotic syndrome, medical management with steroid should be initiated. For patient who failed medical management of nephritic syndrome or with tumor that failed to regress, radiotherapy should be considered. Patient who has severe renal involvement, an aggressive treatment with corticosteroid and cyclophosphamide is advocated. The course of the disease is relatively long and benign, but recurrences are common after surgical excision of the lesion. Eosinophilia is almost always present, although there is no systemic visceral involvement. There have been no reported fatalities $[3,4]$.

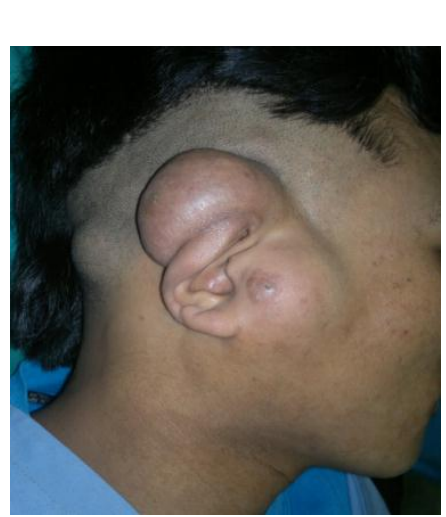

Fig 1: pre-operative

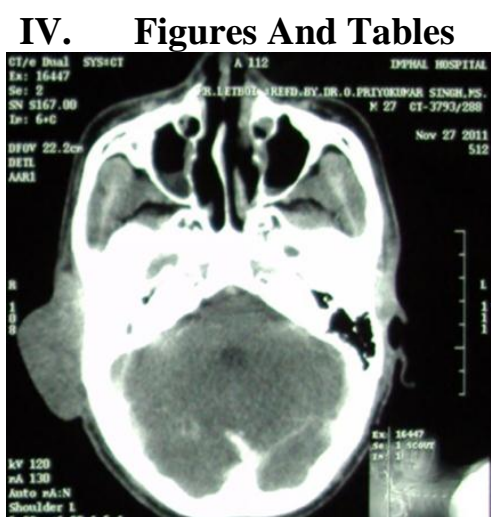

Fig 2: CT scans of PNS

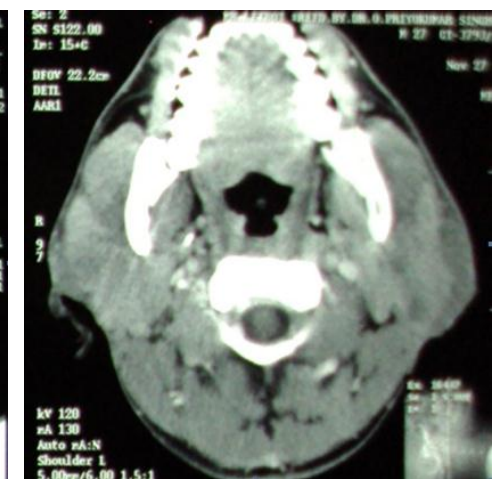

Fig 3: CT scan shows bilateral disease

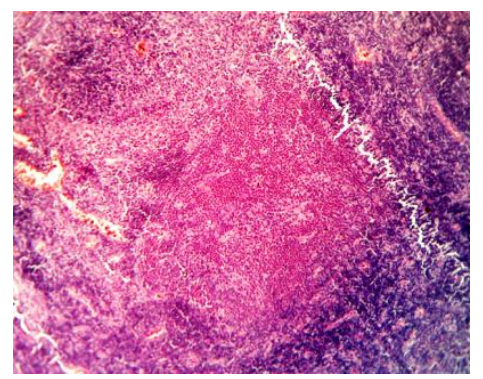

Fig 4: Microphotograph showing prominent eosinophilic microabscess at the interfollicular region (10X)

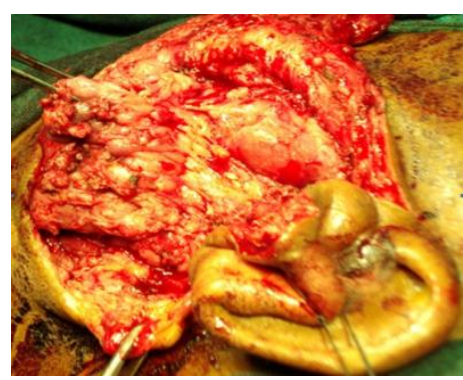

Fig 5: intraoperative picture

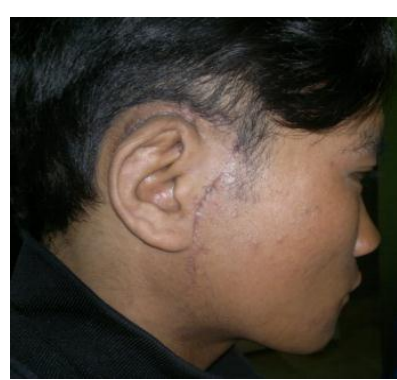

Fig 6: post-operative image

\section{Conclusion}

Kimura's disease is a rare inflammatory disease of unknown etiology and is more common in Asian origin. Among the various manifestations of the disease, involvement of the salivary gland and cervical lymph node is a common occurrence. We report a case of kimura's disease in association with rhinosinusitis, which we find is unique as we found no reported case of association with sinusitis according to our literature search. We also present a short review of literature of the diagnosis and management of this rare disease. With the reporting 
of this case, we attempted to expand the clinical knowledge of Kimura's disease for a better understanding and management.

\section{Acknowledgements}

We are indebted to our teachers who guided us in the diagnosis and treatment of the patient. Thanks are also due to the anaesthetist and staff in the department of Otorhinolaryngology that took part in this study and management of this case.

\section{References}

[1] Hong Chen, Lester D. R. Thompson, Nadine. Kimura Disease A Clinicopathologic Study of 21 Cases. Am J Surg Pathol 2004 Apr ;28(4): 505-13.

[2] Pedro Leonardo Briggs. Kimura disease is not angiolymphoid hyperplasia with eosinophilia: clinical and pathological correlation with literature review and definition of diagnostic criteria. An Bras Dermatol 2006;2:167-73.

[3] A Asma, A Maizaton. Kimura's Disease: An Unusual Cause of Cervical tumour. Med J Malaysia 2005 Aug;60(3):373-76.

[4] Tie-Jun LI, Motoo Kitano. Oriental Kimura's Disease and its Relation to Angiolymphoid Hyperplasia with Eosinophilia (ALHE). South Pacific Study1997;17(2):287-300.

[5] S. R. Ranka, A. Rajput, C. V. Kantharia . Kimura's disease. IJOHNS 2004 January; 56(1):43-45.

[6] Feroze Khan,Sophia Amalanathan, Anand Job, Lisa Choudhrie. Kimura's disease in the external auditory canal. Kimura's disease in the external auditory canal. Otorhinolaryngology and Head \& Neck Surgery, 2011 Aug;8(2):35-36.

[7] ZA Ibrahim, KL Pan, SL Wong, PS Shanmugam, AN Zulkarnaen. Kimura Disease: An Unusual Presentation in Paediatric Age Group. Malaysian Orthopaedic Journal 2011;5(2):54-55.

[8] A. Hafiz, A Yusuf, I.Rosmaliza, N Premchandran, R Kalavathy. Kimura's Disease with Atypical Musculoskeletal Presentation. Malaysian Orthopaedic Journal 2010;4(2):44-47.

[9] H Shahrul, A Baharudin, O Eftat. Kimura's Disease in Malay Patients. Med J Malaysia 2007 Aug ;62(3):263-64. 